\title{
Analysing consumer protection for gamblers across different online gambling operators: A descriptive study
}

\author{
Maris Bonello $^{1,2}$, and Mark D. Griffiths ${ }^{1}$ \\ ${ }^{1}$ Psychology Division, Nottingham Trent University, Nottinghamshire, UK, NG1 4BU \\ ${ }^{2}$ Kindred Group Plc, Fawwara Buildings, Gzira, GZR1405, Malta
}

Keywords Responsible gambling, Social responsibility, Player protection, Responsible gambling tools, Harm minimisation.

\section{Conflicts of Interest}

The first author is an employee of Kindred Group that provides online gambling services. Any online gambling company owned by the Kinded Group was omitted from the evaluation in the present study. The second author has received funding for a number of research projects in the area of gambling education for young people, social responsibility in gambling, and gambling treatment from the Responsibility in Gambling Trust, a charitable body which funds its research programme based on donations from the gambling industry. The second author has also undertaken consultancy for various gaming companies in the area of social responsibility.

\section{Author Contribution Statement}

All authors contributed to the conception and drafting of the paper, revising it critically for important intellectual content, and provided approval for the final version to be published. 


\title{
Analysing consumer protection for gamblers across different online gambling operators: A descriptive study
}

\begin{abstract}
Over the last decade, online gambling has been steadily increasing both in availability and in popularity. Player protection has been in the centre of gambling regulation, and various initiatives have been set in place by some online gambling companies in order to ensure responsible gambling and harm minimisation. The aim of the present exploratory study was to evaluate how online gambling operators protect and minimize harm for their consumers (i.e., their gambling clientele). In order to evaluate the responsible gambling initiatives aiding player protection, the 50 most advertised online gambling websites were examined in relation to their responsible gambling practices (including which responsible gambling tools the operator offered, the presence or absence of a responsible gambling page, problem gambling selfassessment test, age verification procedures, etc.). The findings demonstrated that although most operators engage in at least some responsible gambling practices, there is wide inconsistency amongst different online gambling operators. To evaluate customer service communication, one of the research team posed as a potential problem gambler and collated all verbatim interaction. Again, the findings demonstrated wide inconsistencies between online gambling operators. It is concluded that while some online gambling operators appear to be socially responsible, there are a number of areas where further improvement is needed (e.g., age verification, customer service feedback, direct marketing to players).
\end{abstract}

Keywords Responsible gambling, Social responsibility, Player protection, Responsible gambling tools, Harm minimisation. 


\section{Introduction}

Online gambling has been steadily increasing over the last 15 years ${ }^{1}$. In 2001 , a national prevalence survey in the UK reported only $1 \%$ of over 2000 participants had ever gambled on the internet ${ }^{2}$. In the most recent British Gambling Prevalence Survey, it was reported that $14 \%$ of the nationally representative adult population had gambled online ${ }^{3}$. Concern has been raised about online gambling especially with regards to specific issues that are associated with this activity including availability, accessibility, anonymity, dissociation and disinhibition ${ }^{4-8}$. Online gambling has also been perceived as a safer and more acceptable gambling by specific groups of people such as female gamblers as the online environment is gender-neutral ${ }^{2,5}$. Other positive aspects include the fact that the virtual environment can provide short-term comfort, fun and/or excitement and a haven for distraction ${ }^{9,10}$. Online gambling has also opened new concerns such as the possibility to gamble at work due to easy internet accessibility, which is not easily detected at the workplace ${ }^{11}$. In fact, individuals can now gamble anywhere including at home and on the move due to access via mobile smartphones, tablets, and laptops ${ }^{9}$.

Due to the aforementioned factors, research has shown that problem gamblers are more likely to gamble online ${ }^{9,12,13}$. Online gambling can be riskier for problem gamblers because online gambling offers a function for mood-modifying experiences, including escapism, immersion and dissociation, which may be more prominent in the online gambling world. This excessive involvement can - in some cases - lead to more problematic gambling behaviour ${ }^{8}$. However, because of the way that online companies can collect behavioural tracking data on their clientele, internet gambling may in turn offer possibilities for utilizing responsible gambling (RG) tools (e.g., temporary self-exclusions, personalized behavioural feedback, limit setting tools, pop-up reminders, etc.) that might be difficult in a land-based setting unless player cards are used to track the totality of a gambler's behaviour ${ }^{14}$.

Online gambling involves several stakeholders including gambling operators, community groups, researchers, treatment providers, and the government ${ }^{15}$. Governments play an important role in gambling activity but may have a conflicting position as they often promote it, but at the same time need to be aware of the societal problems it may cause their citizens ${ }^{16}$. Consequently, governments have been slow at recognising corporate social responsibility and the need for RG due to this conflict of interest ${ }^{17}$. In some cases, this conflict of interest is also amplified because the government may be the direct promoter through national lotteries ${ }^{16}$, and 
therefore needs to take note of advertising responsibly and giving information about the risks that come with online gambling in order to counterbalance this conflict of interest ${ }^{18}$.

The regulation of gambling has always been concerned with its social risks with a primary role of consumer protection ${ }^{19}$. Consumer protection is also something that concerns online gambling operators in a commercial manner to protect the company's brand ${ }^{20}$. This has resulted in a topic of great interest in how to engage corporate social responsibility in controversial activities such as online gambling ${ }^{21}$. Therefore, the aforementioned different stakeholders need to acknowledge that there are existing conflicts of interest ${ }^{14}$. To overcome such conflicts of interest, public policies should be based on empirical data and not personal beliefs, and research should be encouraged in co-operation with the gambling operators, despite the possible hindrance the operator may elicit due to the chance of revealing and/or exposing too much about their day-to-day business and practices $^{22}$.

In order to provide safe environments for gamblers (both online and offline), policies should be based on a tripartite model that includes the government, the gambling operators, and those advocating individual harm minimisation ${ }^{14}$. Considering the potential risks of online gambling, the conflicts of interest, and consumer protection, RG should be the highest priority among any gambling operator ${ }^{22}$. Responsible gambling refers to a duty of care including consumer education, playing within limits, information to allow informed choice that includes resources for help, information about games, and information to combat misconceptions and gambling fallacies ${ }^{15}$.

In order to understand the level of these safeguards provided by the gambling industry, Smeaton and Griffiths ${ }^{23}$ examined the RG practices of 30 UK-owned online gambling websites. The results (based on data collected in 2003) showed that there was a lack of RG initiatives at the time. Arguably, online gambling needs more consideration, because in this field of study, geographical boundaries become non-existent and technology may facilitate competition between the gambling providers and regulators ${ }^{19}$, although this might put off some gamblers because the gambling provider is not locally licensed. This lack of geographical boundary and the lack of a strong regulation of online gambling has led to third-party organisations attempting to identify what consumer protection practices are available and what RG practices should be ensured ${ }^{21}$. It has also been argued that there should be an independent authority that can provide enough information to inform legislation and dictate public health initiatives ${ }^{14}$. Given this background, the aim of the present exploratory study is to evaluate how online 
gambling operators protect and minimize harm for their consumers (i.e., their gambling clientele). Details of the specific RG practices investigated are found in the 'Method' section below.

\section{Method}

The present study examined 50 online gambling websites. The selection for the gambling operators chosen was carried out via different online search engines (i.e., Google, Bing, and Yahoo!). The 50 most advertised online gambling operators with a 'dotcom' suffix were chosen (a full list of the websites examined with their online website address can be found in Appendix 1). Due to its 'no-boundaries' nature, online gambling may be provided in countries where gambling has not been regulated and via operating licenses that are not necessarily operating in that country. In fact, a large number of gambling operators are based in Malta and Gibraltar ${ }^{24}$. Each online gambling operator's website was examined in further detail by checking for the following RG practices:

- A dedicated RG page including the following criteria:

- A statement on the operator's commitment to RG

- A warning that gambling can be harmful

- Reference to a problem gambling help organisation and/or self-help groups

○ A self-assessment test for problem gambling

○ Information about the RG tools offered by the operator

○ No promotional gambling material

- Links to gambling filtering software such as GamBlock and/or Betfilter

- Initial age checks during the account registration phase

- A link to the RG page or information about RG practices in the communication sent by the operator to those registering to gamble on the website

- The presence and easy accessibility of gambling account history

- The availability RG tools including:

○ Limit setting facilities

- Cooling off periods

○ Self-exclusion periods

○ Other RG tools

- A link to limit-setting option on the deposit page

- RG-oriented customer service communication 
With regards to the final RG practice in the list, the evaluation was carried out via the customer service communication channel. To assess how RG-oriented the service was, contact was made with customer service representatives using the following two questions and single statement:

1. I would like to control my gambling. Do you have any information on how I can do that?

2. What happens if I increase or remove any of the limits I set?

3. Ifeel a bit addicted sometimes and cannot control my gambling.

These three issues were explored via live chat, and in the cases where this option was not available, email correspondence with the gambling operator was used. Because of the method employed, full transcripts of all the online conversations were obtained.

\section{Results}

The findings were not consistent when looking at the different operators. Some operators demonstrated a high commitment to RG through the information and options available, but other operators lacked these RG initiatives. Evaluation of each of the RG practices listed in the previous section is described below.

Responsible gambling page

Of the 50 online gambling operators, all had a statement on their commitment to RG and all had a warning that gambling could be harmful. Most operators $(n=42)$ referenced an organisation where players could get help for a gambling problem (84\%), and approximately two-thirds of the gambling operators $(n=32)$ displayed a self-assessment test for problem gambling (64\%). Many of the operators $(n=42)$ displayed information about the $R G$ tools that they offered on their website $(84 \%)$. A large majority of the operators $(n=44)$ displayed commercial promotion on the RG page (88\%). One of the gambling operators displayed commercial promotional material on the $\mathrm{RG}$ page via a pop-up window that could not be closed. Approximately two-thirds of the gambling operators $(n=30)$ mentioned or provided links to gambling filtering software to block access from online gambling websites $(60 \%)$.

Age verification check at the account registration stage

When gamblers registered for an account with an online gambling operator, approximately two-thirds of them $(n=34)$ had prominently displayed that the gambling service provided is for 
individuals who are 18 years of age or above (68\%). In no instance were registrants required to provide any identity documents that they were 18 years of age or over (i.e., no age verification checks were carried out by any of the 50 gambling operators).

Responsible gambling information sent to players via email

Almost every operator $(n=47)$ sent an initial email after registration to the players, whether it was a welcome email or an email to encourage depositing (94\%). Of the 47 gambling operators that sent an email after registration, 22 of them (47\%) mentioned RG and/or had a link to the RG page in the email sent. However, in a small number of cases $(n=5 ; 11 \%)$, when the RG link which was communicated in the promotional email was clicked, the link resulted in other areas of the gambling operator website, and not the RG page. As noted in more detail below, the first author explicitly informed the gambling operator about the possibility of having gambling addiction. Responsible gambling operators would be expected to remove those claiming to have a gambling problem from such promotional mailing list, and three-quarters of the operators did so $(n=37 ; 74 \%)$. However, the remainder continued to send commercial emails (between 1 to 12 emails) in the 14 days after the gambling operator had been informed that the first author might have a gambling addiction.

\section{Access to gambling account history}

Most operators $(n=47)$ provided an option for gamblers to have access to their historical gambling data $(\mathrm{n}=94 \%)$.

\section{Responsible gambling tools}

Most operators had RG tools such as limit-setting ( $\mathrm{n}=45$; 90\%), cooling-off period $(\mathrm{n}=36$; $72 \%)$, and voluntary self-exclusion $(n=43 ; 86 \%)$. The limit-setting options were split in the following manner:

- Operators having a deposit limit option only $(n=24 ; 48 \%)$

- Operators having a spending/loss/wager limit only $(n=4 ; 8 \%)$

- Operators having both a deposit limit and the spending limit $(n=13 ; 26 \%)$

- Operators having both a deposit limit and spending limit per product $(n=3 ; 6 \%)$

- Operators having a spending limit per product only $(\mathrm{n}=1 ; 2 \%)$

- Operators having an additional session/time limits $(n=21 ; 42 \%)$ 
Cooling off periods refer to any break available that was less than 6 months $(n=36 ; 72 \%)$, and the voluntary self-exclusion referred to any possible break of six months $(n=43 ; 86 \%)$. There were some operators who offered other RG tools than the ones mentioned above. One operator offered a gaming insurance that said they provided professional support for customers with gambling addiction. Three operators also offered the option to block access to certain products, whereas four operators offered the possibility for gamblers to input details to control their gambling through a form of diary or budget calculator.

Although the option to use RG tools was mentioned on the gambling operators' websites ( $\mathrm{n}=42$; $84 \%$ ), this was not always truthful. In one case, it was noted in the small print that a selfexclusion request might take up to 48 hours to be processed. Twelve operators mentioned that in order to self-exclude, the gambler had to contact customer services and/or fill in a form to send via email. In one instance, all the information concerning possible RG tools was only accessible via customer services, and in another case, gamblers did not have access to the RG tools unless real money was deposited on their account. In some cases, the information about the RG tools was not encouraging RG practice. For example, in one instance, the following sentence was present when accessing the self-exclusion option: "Customers who block their account will no longer be able to deposit funds into their account or take advantage of our offers."

\section{Interactions with customer service}

In order to determine first-hand the RG practice involved with each gambling operator, two questions and one statement were presented to the customer service team (see 'Method' section). The preferred method to present these concerns by the first author was via a live online chat facility, and when this was not readily available, the questions were asked via emailing customer services. Almost three-quarters of the operators $(n=35)$ offered the option of live chat $(70 \%)$, with the remaining operators $(n=15)$ being contacted via email $(30 \%)$.

The first representation of the results is presented based on the live chat transcripts. With regards to the first question asked (I would like to control my gambling. Do you have any information on how I can do that?), 30 out of 35 operators (86\%) suggested or provided links to the functionality of limit setting. One interaction involved probing for more detail, another interaction ignored the question asked, and three of the interactions suggested voluntary selfexclusion. In answer to the second question (What happens if I increase or remove any of the limits I would set?), eight customer service interactions mentioned 24-hour waiting period 
whereas 14 mentioned a seven-day waiting period. Five customer service interactions ignored the question and did not answer it, whereas six showed a lack of knowledge and/or misunderstanding. In one instance, the first author was told that there needed to be a discussion with the gambling operator's account manager.

With regards to the last statement presented to the customer service agent ( I feel a bit addicted sometimes and cannot control my gambling), the answers varied from being given access to a self-exclusion form to miscommunication. The responses were categorised into three different headings; (i) suggestions concerning RG ( $\mathrm{n}=17 ; 49 \%)$, (ii) performing an action $(\mathrm{n}=8 ; 23 \%)$, and (iii) bad practice $(\mathrm{n}=10 ; 29 \%)$. Of 35 operators responding via an online live chat facility, 17 operators (49\%) suggested an RG measure including checking information on RG, voluntary self-exclusion, and doing a problem gambling self-assessment test. Eight gambling operators performed a specific action (e.g., closing of the account) without the agreement of the first author (23\%). Ten operators engaged in bad practice $(29 \%)$. In three cases, the information given was irrelevant for someone who was going through a problem, such as information about the designated account manager, queries about Malta, and the fact that the customer service agent did not like gambling because gambling was equated with addiction. In six cases, the remarks were completely ignored and the online interaction was ceased by the gambling operator. In one case, a bonus was offered to the first author to continue gambling. After the live online chat interaction, seven operators contacted the first author with more information about RG tools via email, while six of the operators closed the account without any choice for the gambler.

For the other 15 operators where emails were sent, six of them closed the account without providing any other choice, five gave generic information about RG tools, and four operators did not provide a reply - just an acknowledgement of the email sent. In most cases, the reply to the email was done either on that day or the next day, but in one case, the email was answered after 13 days. Finally, the number of gambling operators that allowed access to the account after the information about having a gambling addiction was given to the customer service representative was examined. Over half of the operators $(n=28)$ still allowed account access $(56 \%)$ whereas the remainder $\operatorname{did} \operatorname{not}(\mathrm{n}=22 ; 44 \%)$. One month after the communication with customer services, 26 operators allowed account access whereas 24 operators did not.

\section{Discussion}


The aim of the present study was to evaluate different online gambling operators and their responsible gambling $(\mathrm{RG})$ practices in protecting their customers. The main findings showed that RG information is consistent across most of the online gambling operators examined, and more than half of them (68\%) displayed information about the 18+ years age restriction at the account registration phase. All gambling operators sent players commercial communication via email upon registration, but only $47 \%$ of the emails actually contained any information about RG. There were quite a few inconsistencies in the RG tools offered, and this inconsistency was also evident in the communication between the customer service department of each operator and the first author. Inconsistencies were also observed in the follow-up after the first author informed the gambling operator that they had a gambling problem during the communication with operator's customer service agents.

An area that appeared to be consistent with most of the gambling operators was the information available in the designated RG page. This page contained information about the operator's commitment to $\mathrm{RG}$, a warning that gambling can be harmful, and a reference to a problem gambling help organisation. The self-assessment test for problem gambling was present in nearly two-thirds of the operators' RG page (64\%). A paradoxical issue was observed in most of the operators' RG page. In $88 \%$ of the gambling operators examined, there was some type of commercial advertisement (typically promoting some type of bonus). The operator should always inform the consumer concerning the potential risks and hazards associated with excessive gambling in order to help enable behavioural change ${ }^{25}$. An RG page on a gambling operator's website should be the first place where consumers resort for advice about RG. If these RG-designated pages also contain promotional information, the purpose of the page is somewhat defeated.

Another area that evidently needed more improvement is age verification of the registered players. The only evidence that the gambling operators took age restriction seriously was in providing a message upon registration that players needed to be aged $18+$ years to gamble. It was difficult to determine whether an age verification check had been carried out by the operator because age verification can take a variety of forms without the knowledge of the consumer, such as cross-referencing the customer details with official data sources ${ }^{26}$. Age verification has been an issue that governments have faced when wishing to liberalise gambling because solutions are needed to protect underage players ${ }^{20}$. It is easy to shift the blame and all responsibility onto the gambling operator, but there are clearly limitations on how much the operator can or may do. It is not the intention of the gambling operator to have underage 
customers as this might cause reputational and integrity issues for the operator in the long-term. When an operator accepts financial transactions from an underage gambler, there are financial consequences for the operator as well, as the operator is obliged to give back the money to the legal guardian of the underage customer ${ }^{27}$. When it comes to Maltese gambling regulations, any deposited funds by underage consumers need to be forfeited to the gambling authority ${ }^{28}$.

Upon registration it was common for all operators to send email communication with specific offers and information about bonuses. It was noted that out of all the communication sent by the operators, only $47 \%$ had an RG message in their email communication. Still, the main area of concern was what happened after the first author admitted to having a gambling problem with each online gambling operator. Despite having disclosed this information, $26 \%$ of the gambling operators still sent promotional and marketing communication. Although the percentage was modest, the impact of sending marketing communication to a consumer who specifically tells the gambling operator that they have a gambling problem may have a negative impact on the individual. In a qualitative research study by Binde ${ }^{29}$, the impact of gambling advertisement on problem gamblers was explored. Several of the participants in this study described how gambling advertisements made it harder to resist the impulse to relapse. Receiving such advertisements directly into the player's personal email inbox is therefore an issue that some operators appear to need educating about.

Another area examined was the facility of using various RG tools across the 50 operators. According to Auer and Griffiths ${ }^{29}$, voluntary time and money limits are becoming widespread among online gambling operators, but what was evident in the present study's evaluation was that although these tools are available, they might not always be available in the most convenient and user-friendly manner. The online gambler can do several things on their account, such as easily deposit money, play on a variety of games, and withdraw money back to their payment method. Despite the technological advancements that have made this possible, 12 gambling operators only offered RG tools via an email request (that may result in delayed request processing or a request not being processed at all).

Online gambling operators have the capability to provide a variety of limit types such as deposit, play, loss and bet, and these may differ from being fixed (which are easier to administer but place everyone's disposable income on one scale) or voluntary (which supports informed adult choice $)^{13}$. There is a lack of evaluative empirical research on $\mathrm{RG}$ tools ${ }^{30}$, and this might be the reason for certain inconsistencies between different gambling regulations. Looking into 
the discrepancy between the operators in the present study, there might be a question with regards to how empirically-based these legal requirements were. Arguably, Wood and colleagues $^{31}$ carried out an example of good research examining RG practices. In their study, RG experts, treatment providers, and recovered problem gamblers were asked on the perceived effectiveness of 45 different RG tools. The highly recommended RG features for online gambling included cash payment outside of the online gambling session, player-initiated temporary or permanent self-exclusion, spending limits, and a 24-hour 'panic' button (which by pressing, instantly closes down the gambling session). Such research should be encouraged in order to provide online RG tools that are empirically proven to help gamblers and not based on a hunch and/or what is based on perceived good practice.

One RG tool that appears to be popular amongst gamblers is the temporary self-exclusion option, where consumers can choose to temporarily remove access to their online gambling account. In research by Griffiths and colleagues ${ }^{32}, 46 \%$ of their participants (over 2500 online gamblers) reported that the 7-day self-exclusion was a useful RG tool. This was followed by the 1-month self-exclusion, and the daily self-exclusion option. Among the 50 online operators evaluated in the present study, $72 \%$ of the operators had an option for a self-exclusion that was less than six months, and $86 \%$ offered six-month self-exclusion. Online self-exclusion has its advantages when compared to land-based self-exclusion as it only requires a few clicks with low access barriers ${ }^{33}$, and when provided online. In fact, not offering temporary self-exclusion could be argued to be the same thing as encouraging further play ${ }^{23}$. More importance should be given in order to make sure that all online operators provide such an important and popular RG tool.

A potential cause for concern was the interaction with customer services and the promotion of RG through this medium. For all 50 gambling operators, there was a possibility of contacting customer service for help. This may be a common occurrence for customers to contact customer service when assistance is needed even with RG $\operatorname{cases}^{34}$. The biggest issue is the lack of consistency concerning the information provided to the gambler. Moreover, some of the information given was arguably unethical and/or might cause further issues with gambling for that individual. As mentioned above, when one gambling operator was specifically informed that the consumer was experiencing problem gambling issues, a bonus was offered to continue gambling. 
This type of practice is evidence of bad practice but it may be that there is a lack of training on these RG practices at an operator level. Training employees concerning RG will help employees in their daily jobs and provide the employee with better information in referring a distressed gambler ${ }^{35}$. Although research concerning employees in the gambling industry and RG training may be scarce, it has been shown that RG training is associated with an increased knowledge on the topic ${ }^{36}$. Management involved in the organisation of training for gambling operator employees also need to consider the frequency needed for the training, as certain gains achieved from training may not be maintained after six months ${ }^{35}$.

Although the present study was beneficial in examining which RG initiatives are being offered across different online operators, this study had a number of limitations. The main shortcomings were that only a limited number of online gambling websites were examined $(n=50)$ and that RG-oriented communication was based on only one customer service interaction. However, it is worth noting that the sample chosen included some of the biggest and best known online gambling operators in the world. Suggestions for future study include replication of the present study with a larger selection of gambling websites and carried out over a longer period in order to determine whether RG initiatives change and develop across different online gambling operators. Another suggestion would be to replicate the study to compare RG initiatives with locally licensed online gambling websites, or a study including interviews with the operators' representatives themselves.

Although the operators sampled was small, a number of recommendations can be made. All operators, irrespective of size or market share should provide (i) age verification checks to prevent minors gambling, (ii) signposting towards a referral service to help those who think they may have a gambling problem, (iii) blocking software for those gamblers that request it, (iv) information about how to access all responsible gambling tools, (v) high ethical standards (for instance, companies should not be sending marketing and promotional materials to those customers that have admitted they have a gambling problem or including promotional material on responsible gambling webpages), (vi) an easy to use voluntary self-exclusion system that does not involve contacting customer services or filling out a long form, and (vi) training to customer service agents so that they can deal with issues and queries from problem gamblers in a knowledgeable, helpful, and informative manner.

\section{Conclusion}


While some online gambling operators appear to be socially responsible, there are a number of areas where further improvement is needed (e.g., age verification, customer service feedback, direct marketing to players). Overall, it is likely that online gambling is (and will continue to be) an activity that will increase in participation. Therefore, RG practices are critical in order to ensure consumer protection. Thus, a strategic framework is needed to establish responsible gambling oriented policies based on empirical studies that in turn will reduce any possible socio-political influences $^{22}$. This is also in the interest of the gaming operators as a lack in trust and credibility will in turn create a commercial disadvantage ${ }^{37,38}$.

\section{References}

(1) N. Canale, M.D., Griffiths, A. Vieno, V. Siciliano and S. Molinaro, Impact of internet gambling on problem gambling among adolescents in Italy: Findings from a large-scale nationally representative survey, 57 COMPUTERS IN HUMAN BEH. 99-106 (2016).

(2) H. Wardle, A. Moody, S. Spence, J. Orford, R. Volberg, D. Jotangia, M.D. Griffiths, D. Hussey and F. Dobbie, BRITISH GAMBLING PREVALENCE SURVEY 2010. London: The Stationery Office (2011).

(3) M.D. Griffiths, Internet gambling: Preliminary results of the first UK prevalence study, 5 J GAMBLING ISSUES (2001), <http://jgi.camh.net/doi/full/10.4309/jgi.2001.5.8> (last accessed Nov. 30, 2016).

(4) A. McCormack and M.D. Griffiths, A scoping study of the structural and situational characteristics of internet gambling, 3(1) INT'L J CYBER BEHAVIOR, PSYCHOLOGY AND LEARNING. 29-49 (2013).

(5) R. Corney and J. Davis, The attractions and risks of Internet gambling for women: A qualitative study, 24 J GAMBLING ISSUES, 121-139 (2010).

(6) A.D. Smith and W.T. Rupp, Service marketing aspects associated with the allure of egambling, 26(3) SERVICES MARKETING QUARTERLY. 83-103 (2005).

(7) J. Suler, The online disinhibition effect, 7 CYBERPSYCHOLOGY AND BEHAVIOUR. 321-326 (2004).

(8) M.D. Griffiths, Internet gambling: Issues, concerns and recommendations, 6 CYBERPSYCHOLOGY AND BEHAVIOR. 557-568 (2013).

(9) D.J. Kuss and M.D. Griffiths, Internet gambling behaviour, in ENCYCLOPEDIA OF CYBER BEHAVIOR 735-753 (2012). 

“pathological” subject, 51 AMERICAN BEHAVIORAL SCIENTIST. 33-55. (2007).

(11) M.D. Griffiths, Internet gambling in the workplace, $21 \mathrm{~J}$ WORKPLACE LEARNING. 658-670 (2009).

J. McBride and J. Derevensky, Internet gambling behavior in a sample of online gamblers. 7 INT'L J MENTAL HEALTH AND ADDICTION. 149-167 (2009).

M.D. Griffiths and A. Barnes, Internet gambling: An online empirical study among student gamblers, 6 INT'L J MENTAL HEALTH AND ADDICTION. 194-204 (2008)

(14) R.T.A. Wood and M.D. Griffiths, Social responsibility in online gambling: Voluntary limit setting, 9(11) WORLD ONLINE GAMBLING LAW REPORT. 10-11 (2010).

A. Blaszczynski, P. Collins, P., D. Fong, R. Ladouceur, L. Nower, L., H.J. Shaffer and J.L. Venisse, Responsible gambling: General principles and minimal requirements, 27 J GAMBLING STUD. 565-573 (2011).

(16) J. Orford, Governments as promoters of dangerous consumptions, 104 ADDICTION. 693-695 (2009).

L. Hancock, T. Schellinck and T. Schrans, Gambling and corporate social responsibility (CSR): Re-defining industry and state roles on duty of care, host responsibility and risk management, 27 POLICY AND SOCIETY. 55-68 (2008).

R.T. Wood and R.J. Williams, Problem gambling on the Internet: Implications for Internet gambling policy in North America, 9 NEW MEDIA AND SOCIETY. 520542. (2007).

(19) J.P. Vileneuve, Gambling regulation and risk, 1 EUROPEAN J RISK REGULATION. 415-418 (2010).

(20) M. Carran, Minors and gambling regulation, 4 EUROPEAN J RISK REGULATION. 509-520 (2013)

(21) Y. Cai, H. Jo and C. Pan, Doing well while doing bad? CSR in controversial industry sectors, 108 J BUSINESS ETHICS, 467-480 (2012).

(22) A. Blaszczynski, R. Ladouceur and H.J. Shaffer, Science-based framework for responsible gambling: The Reno model, 20 J GAMBLING STUDIES. 301-317 (2004).

(23) M. Smeaton and M.D. Griffiths, Internet gambling and social responsibility: An exploratory study. 7 CYBERPSYCHOLOGY AND BEHAVIOR. 49-57 (2004). 
M. Auer and M.D. Griffiths, Behavioral tracking tools, regulation, and corporate social responsibility in online gambling, 17 GAMING L. REV \& ECON. 579-583 (2013).

(25) S.M. Monaghan and A. Blaszczynski, Electronic gaming machine warning messages: information versus self-evaluation, $144 \mathrm{~J}$ PSYCHOLOGY: INTERDISCIPLINARY AND APPLIED. 83-96 (2009).

(26) M.D. Griffiths and J. Parke, Adolescent gambling on the Internet: A review, 22(1) INT’L J ADOLESCENT MEDICINE AND HEALTH. 59-75 (2010).

<http://www.ecogra.org/downloads/eCOGRA_Generally_Accepted_Practices_(eGAP ..pdf> (last accessed Nov. 30, 2016).

(28) Remote Gaming Regulations. SUBSIDIARY LEGISLATION 438.04 OF LOTTERIES AND OTHER GAMES ACT, Malta (2004).

(29) P. Binde, Exploring the impact of gambling advertising: An interview study of problem gamblers, 7 INT'L J MENTAL HEALTH AND ADDICTION, 541-554 (2009).

(30) M. Auer and M.D. Griffiths, Voluntary limit setting and player choice in most intense online gamblers: An empirical study of gambling behaviour, 29 J GAMBLING STUDIES. 647-660 (2013).

(31) R.T.A. Wood, G. Shorter and M.D. Griffiths, Rating the suitability of responsible gambling features for specific game types: A resource for optimizing responsible gambling strategy, 12 INT'L J MENTAL HEALTH AND ADDICTION. 94-112 (2014).

M.D. Griffiths, R.T. Wood and J. Parke, Social responsibility tools in online gambling: A survey of attitudes and behavior among internet gamblers, 12 CYBERPSYCHOLOGY AND BEHAVIOR. 413-421 (2009).

T. Hayer and G. Meyer, Internet self-exclusion: Characteristics of self-excluded gamblers and preliminary evidence for its effectiveness, 9 INT'L J MENTAL HEALTH AND ADDICTION. 296-307 (2011).

(34) J. Haefeli, J., Lischer and J. Schwarz, Early detection items and responsible gambling features for online gambling, 11 INT'L GAMBLING STUDIES. 273-288 (2011). 

training program on responsible gambling for casino employees, 6 INT'L J MENTAL HEALTH AND ADDICTION. 594-601 (2008).

(36) D.A. LaPlante, H.M. Gray, R. LaBrie, J.H. Kleschinsky and H.J. Shaffer, Gaming industry employees' responses to responsible gambling training: A public health imperative, 28 J GAMBLING STUDIES. 171-191 (2012).

(37) S. Gainsbury, J. Parke and N. Suhonen, Consumer attitudes towards Internet gambling: Perceptions of responsible gambling policies, consumer protection, and regulation of online gambling sites, 29 COMPUTERS IN HUMAN BEH. 235-245 (2013).

(38) Wood, R.T.A. \& Griffiths. M.D. Why Swedish people play online poker and factors that can increase or decrease trust in poker websites: A qualitative investigation, 21 J GAMBLING ISSUES. 80-97 (2008). 
Appendix 1: List of online gambling websites examined in the present study

\begin{tabular}{|c|c|}
\hline Website & Website domain \\
\hline 32red & www.32red.com \\
\hline 7red & $\overline{\text { www.7red.com }}$ \\
\hline 888 & www.888.com \\
\hline Bet-at-home & www.bet-at-home.com \\
\hline Bet365 & www.bet365.com \\
\hline Betfair & $\overline{\text { Www.betfair.com }}$ \\
\hline Betfred & www.betfred.com \\
\hline Betsafe & www.betsafe.com \\
\hline Betsson & www.betsson.com \\
\hline Bingo for money & www.bingoformoney.ag \\
\hline Break the Bank Bingo & www.breakthebankbingo.com \\
\hline Bwin & www.bwin.com \\
\hline Casino Euro & Www.casinoeuro.com \\
\hline Cheeky Bingo & www.cheekybingo.com \\
\hline Cherry Casino & www.cherrycasino.com \\
\hline Come On & www.comeon.com \\
\hline Coral & www.coral.co.uk \\
\hline Costa Bingo & $\overline{\text { Www.costabingo.com }}$ \\
\hline Dotty Bingo & www.dottybingo.com \\
\hline EuroCasinoBet & www.eurocasinobet.com \\
\hline Euro Grand & $\begin{array}{l}\text { www.eurogrand.com } \\
\end{array}$ \\
\hline Europa Casino & www.europacasino.com \\
\hline Expekt & en.expekt.com \\
\hline Foxy Bingo & www.foxybingo.com \\
\hline Gala Casino & Www.galacasino.com \\
\hline Guts & Www.guts.com \\
\hline Inter Casino & www.intercasino.com \\
\hline Ladbrokes & 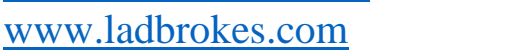 \\
\hline Leo Vegas & www.leovegas.com \\
\hline Little Miss Bingo & www.littlemissbingo.com \\
\hline Lucky Red Casino & www.luckyredcasino.com \\
\hline Mansion & WwW.mansion.com \\
\hline Casino Club & Www.casinoclub.com \\
\hline mFortune & www.mfortune.co.uk \\
\hline Moon Bingo & Www.moonbingo.com \\
\hline Mr Green & www.mrgreen.com \\
\hline Nordic Bet & www.nordicbet.com \\
\hline Paddy Power & www.paddypower.com \\
\hline Paf & www.paf.com \\
\hline Party Casino & casino.partycasino.com/en/ \\
\hline Poker Stars & www.pokerstars.com \\
\hline Polo Bingo & WwW.polobingo.com \\
\hline Redbet & www.redbet.com \\
\hline Safer Gambling & www.safergambling.com \\
\hline Sporting Bet & www.sportingbet.com \\
\hline Super Casino & Www.supercasino.com \\
\hline Tipico & Www.tipico.com \\
\hline
\end{tabular}


TitanBet

William Hill

Wow Bingo www.titanbet.com

www.williamhill.com

www.wowbingo.co.uk 